Table 1. Study Population Characteristics

\begin{tabular}{|c|c|c|c|}
\hline \multirow[b]{2}{*}{ Age (years) mean $\pm \mathrm{SD}$} & \multicolumn{3}{|c|}{ AxSpa n:204 Controls n:106 p value } \\
\hline & $43.1 \pm 11.4$ & $40.6 \pm 12.6$ & $>0.05$ \\
\hline Male $n(\%)$ & $125(61.3)$ & $26(24.5)$ & $<0.001$ \\
\hline Education time (years) mean $\pm \mathrm{SD}$ & $9.8 \pm 4.3$ & $9.7 \pm 4.0$ & $>0.05$ \\
\hline Current smoker $\mathrm{n}(\%)$ & $77(37.7)$ & $31(29.2)$ & $>0.05$ \\
\hline Alcohol consumption $\mathrm{n}(\%)$ & $60(29.4)$ & $9(8.5)$ & $<0.001$ \\
\hline Current BMI kg/m² mean \pm SD & $26.8 \pm 4.6$ & $26.5 \pm 4.7$ & $>0.05$ \\
\hline Weight gain group $n(\%)$ & $62(30.4)$ & $40(37.7)$ & $>0.05$ \\
\hline Weight stable group n(\%) & $142(69.6)$ & $66(62.3)$ & \\
\hline Current BASDAI mean \pm SD & $1.8 \pm 1.5$ & N/A & \\
\hline Current BASFI mean \pm SD & $1.5 \pm 1.8$ & N/A & \\
\hline Patients treated with biologic drugs $\mathrm{n}(\%)$ & $118(57.8)$ & $\mathrm{N} / \mathrm{A}$ & \\
\hline Patients treated with conventional drugs $n(\%)$ & $84(41.1)$ & $\mathrm{N} / \mathrm{A}$ & \\
\hline Presence of Anxiety n(\%) & $82(40.2)$ & $34(32.1)$ & $>0.05$ \\
\hline Presence of Depression $n(\%)$ & $89(43.6)$ & $30(28.0)$ & $<0.001$ \\
\hline TFEQ-R21emotional eating mean \pm SD & $5.2 \pm 3.1$ & $5.6 \pm 2.7$ & $<0.05$ \\
\hline TFEQ-R21 uncontrolled eating mean \pm SD & $14.7 \pm 6.3$ & $17.5 \pm 5.4$ & $<0.001$ \\
\hline TFEQ-R21 cognitive restraint mean \pm SD & $14.3 \pm 4.3$ & $15 \pm 4.2$ & $>0.05$ \\
\hline Stable physical activity $\mathrm{n}(\%)$ & $117(57.4)$ & $49(46.2)$ & $>0.05$ \\
\hline Decreased physical activity $n(\%)$ & $87(42.6)$ & $57(53.8)$ & \\
\hline
\end{tabular}

Acknowledgements: Special thanks to our clinical nurse Alev Vayni for her devoted assistance in interviewing patients to fill out the questionaire.

Disclosure of Interests: None declared.

DOI: 10.1136/annrheumdis-2021-eular.592

\section{POS1166 UNDERSTANDING PRESCRIPTION BEHAVIOR ACROSS HEALTHCARE PROFESSIONALS IN TREATING KNEE OSTEOARTHRITIS BEFORE AND DURING THE 2020 COVID-19 PANDEMIC}

D. Baldock ${ }^{1}$, E. Baynton ${ }^{1}$, H. Hamdan ${ }^{2} .{ }^{1} I$ psos MORI, Healthcare Service Line, Iondon, United Kingdom; ${ }^{2}$ Ipsos Sdn Bhd, Healthcare Service Line, Kuala Lumpur, Malaysia

Background: Knee Osteoarthritis (OA) treatment aims to provide symptom relief, easing joint pain and enabling mobility. The COVID-19 pandemic affected healthcare systems globally, including ways OA patients were treated.

Objectives: This study is to understand how treatment management of knee OA patients and prescribing behavior across US healthcare professionals (HCPs) was affected when COVID-19 impacted healthcare systems and regimes globally

Methods: A multi-center online medical chart review study of patients with OA was conducted between May - July 2019 \& 2020 among US rheumatologists (rheums), orthopedic surgeons (orthos), primary care physicians with a focus in sports medicine (SM PCPs), and pain specialists, practicing across hospital and private practices. Recruited from a large access panel, physicians were screened for duration of practice in their specialty (3-50 years) and caseload (35 or more knee OA patients personally managed, at least 10 of which must be classified as moderate-severe). Patient charts were recorded for the next 5 eligible patients seen during the screening period. Respondents abstracted patient demographics and treatment regimes used. Descriptive statistics were used to analyse the data

Results: 275 physicians were recruited and collectively reported 1375 patients between May-Jul 2019; 260 physicians were recruited and collectively reported 1300 patients between May-Jul 2020

Looking at treatments patients were receiving at time of reporting, oral medications, particularly NSAIDs, were widely used, regardless of the physician specialty. A directional increase of $6 \%$ in the proportion of reported patients deriving from sampled primary care SM PCPs from pre-COVID (Q219) to during COVID (Q220), whilst the same is not true for rheums, orthos, and pain specialists.

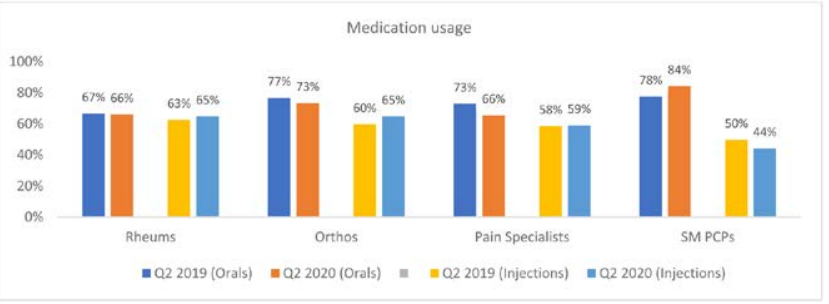

Figure 1. Medication usage in reported knee OA patients across all sampled specialties - Q2 2019 vs Q2 2020

With regards to the usage of injectable therapy, the opposite pattern is seen higher proportions of reported patients deriving from sampled rheums, orthos \& pain specialists receiving injectables vs those deriving from sampled SM PCPs. While focusing on orthos, the proportion of reported patients deriving from them who receive injectables grew by $5 \%$ in Q220 (during COVID) vs Q219 (preCOVID) - directionally more so than other specialties. This is possibly due to limited elective surgeries during the pandemic.

Looking deeper into injectable usage amongst reported patients deriving from sampled rheums and orthos, the proportion of the total reported patient set receiving this treatment type is relatively similar across both specialties, prior to, and during the COVID-19 pandemic. However, among reported patients who received surgery, a directionally higher proportion of this reported patient cohort deriving from orthos are noted to receive injectables vs those from rheums - this is true both pre- and during the pandemic.

Conclusion: From the sample surveyed, it appears that primary care SM PCPS adopted their treatment adjustment during COVID-19 pandemic differently, by prescribing more orals, while other secondary care physicians (rheums, orthos, pain specialists) focused on injection treatment for longer pain relief. Considering movement restrictions and limited elective surgeries during the pandemic, the lack of targeted treatment options for knee OA has been made more apparent. With a burgeoning pipeline, many of which are targeted therapies, it is hopeful that knee OA treaters will be less reliant on symptom remedies and can readily offer patients' longer-acting pain relief or disease modifying options to combat joint deterioration

\section{REFERENCES:}

[1] Ipsos Osteoarthritis Therapy Monitor (May - July 2019, 275 specialists reporting on 1375 OA patients seen in consultation; May - July 2020, 260 specialists reporting on 1300 OA patients seen in consultation, data collected online. Participating physicians were primary treaters and saw a minimum number of 35 knee OA patients). Data (C) Ipsos 2021, all rights reserved.

Disclosure of Interests: None declared.

DOI: 10.1136/annrheumdis-2021-eular.629

\section{POS1167 \\ REVIEW OF THE IMPACT OF COVID-19 PANDEMIC ON RHEUMATOLOGY SERVICES AT A LARGE TERTIARY CARE CENTRE IN WALES, UK}

$\underline{\text { R. Asif }}^{1}$, N. Elndari ${ }^{2}$, A. Negi ${ }^{1}{ }^{1}$ Cardiff and Vale University Health Board, Rheumatology, Cardiff, United Kingdom; ${ }^{2}$ Cardiff and Vale University Health Board, Internal Medicine, Cardiff, United Kingdom

Background: COVID-19 pandemic had an unprecedented impact on the delivery of patient care. Rheumatology services had to rapidly adapt to virtual consultations at the onset of the pandemic. However, providing a high quality and effective service in a virtual setting can be challenging and therefore its prudent to do a formal review and gain patient feedback to ensure that these clinics are fit for purpose.

Objectives: To evaluate the impact of first wave of COVID-19 pandemic on patients with autoimmune rheumatic conditions, assess delivery of rheumatology outpatient care and record patient feedback.

Methods: This study included patients on the Rheumatology clinic lists between $3^{\text {rd }}$ and $31^{\text {st }}$ August 2020. An electronic survey questionnaire was developed and the survey link was sent to patients via a text message using secure IT platform. Data was collected on patient demographics, diagnosis, comorbidities, treatments, clinical/ laboratory confirmed COVID-19 diagnosis, treatment interruption, impact on work, personal protective measures taken and views on virtual consultations.

Results: 307 patients responded with 287 complete responses. $73.1 \%(223)$ were female and $32.4 \%(99)$ were $>65$ years of age. Rheumatoid arthritis was the most common diagnosis $41.6 \%(127)$. Hypertension was the commonest comorbidity $21.4 \%(64)$ followed by Chronic lung disease $17.3 \%(52) .28 .8 \%(85)$ were on Hydroxychloroquine, $26.7 \%(79)$ Methotrexate, $14.2 \%(42)$ Sulfasalazine and $13.2 \%(39)$ on Prednisolone. $22.3 \%(66)$ were on Biologics: Anti TNF $12.8 \%(38)$, Tocilizumab 3.7\%(11) and Rituximab 3\%(9). 52.6\%(161) shielded, 16.9\%(55) self-isolated and $30.3 \%(93)$ only maintained social distance. 197 patients self reported as being vulnerable but based on their treatment,only 167 patiemts me the clinically extremely vulnerable (CEV) criteria and all of those received government shielding letter.

$3.6 \%(11)$ had lab confirmed COVID-19, 3.2\%(10) had clinically suspected COVID-19 infection. $14.3 \%$ (43) had their treatment interrupted. $4.6 \%(14)$ were unable to work from home or maintain social distancing at work. 59.8\%(182) had face-to-face consultation changed to virtual. $63.2 \%(189)$ were satisfied, $28 \%(84)$ neutral and $8.7 \%(26)$ reported dissatisfaction with their consultation. 50.5\%(153) were happy to continue with virtual consultation but with an option of face to face only if necessary. For consultations post COVID-19, 59.4\%(182) preferred a mixture of face to face and virtual appointments.

Conclusion: Majority of our patients seem happy with virtual consultations as long as they are assured of a face-to-face consultation if needed. A minority $(8.7 \%)$ however, were dissatisfied. Some of the suggestions were 
use of video consultations and improvement in communication before the virtual appointments. Our survey also shows that our patients have adapted well to virtual consultations and many are keen to have virtual consultation in the longer term. In our survey, only $6.8 \%(21)$ patients reported definite or clinically suspected COVID-19. Possible explanations for this include strict compliance with government advice on social distancing/shielding and limited testing at the onset of the pandemic. More patients assumed themselves to be clinically CEV than those who were actually CEV based on their treatment which is not surprising because of high level of anxiety among patients due to rapidly spreading pandemic and multiple sources of information.

This feedback provides useful data which will help us to plan the delivery of rheumatology services post COVID-19 pandemic. While face-to-face patient contact is needed for comprehensive disease assessment, teaching and training, a model for the future is likely to include a combination of face-to-face and virtual consultations. This could allow a greater capacity to see new patients and reduce waiting lists. Patients with uncomplicated and stable disease could be followed up in virtual clinics. There is also a need to formally incorportate the virtual consultations into the curriculum for Rheumatology trainees.

Disclosure of Interests: None declared.

DOI: 10.1136/annrheumdis-2021-eular.649

\section{POS1168 SELF-REPORTED SARS-CoV2 TESTING AND COVID-19 DISEASE IN PATIENTS WITH RHEUMATOID ARTHRITIS, AXIAL SPONDYLOARTHRITIS AND PSORIATIC ARTHRITIS IN A SWISS OBSERVATIONAL COHORT}

E. Papagiannoulis ${ }^{1}$, A. Ciurea ${ }^{2}$, D. Dan ${ }^{3}$, A. Finckh ${ }^{4,5}$, B. Gilbert ${ }^{4,5}$, I. Von Loga $^{1}$, C. Melong Pianta Taleng ${ }^{4,5}$, A. Scherer ${ }^{1}$, K. Lauper ${ }^{4,5,6} .{ }^{1}$ SCQM, Statistics, Zurich, Switzerland; ${ }^{2}$ Zurich University Hospital, University of Zurich, Rheumatology, Zurich, Switzerland ${ }^{3} \mathrm{CHUV}$, Rheumatology, Lausanne, Switzerland; ${ }^{4}$ Faculty of Medicine, University of Geneva, Internal Medicine, Geneva, Switzerland; ${ }^{5}$ Hôpitaux Universitaires de Genève (HUG), Rheumatology, Geneva, Switzerland; ${ }^{6}$ Centre for Musculoskeletal Research, University of Manchester, Centre for Epidemiology Versus Arthritis, Manchester, United Kingdom

Background: Since the beginning of the pandemic in Switzerland, immunosuppressed people were strongly advised to be tested for SARS-CoV2 when symptomatic as it was conjectured that they might be more at risk for infection and/ or severe disease. While patients with autoimmune diseases might be indeed more at risk of death from COVID-19 ${ }^{1}$, it remains unknown, whether there are differences in infection or complication rates between patients with rheumatoid arthritis (RA), psoriatic arthritis (PsA) and axial spondyloarthritis ( $\mathrm{AxSpA})$, and whether this relates to their disease or their treatment. Additionally, the prevalence of SARS-CoV2 testing in this population is not known.

Objectives: This study aimed to assess and compare the rate of COVID-19 and SARS-CoV2 testing in patients with RA, AxSpA and PsA, the potential association with their treatment and, for testing, the number of symptoms.

Methods: We included patients with RA, AxSpA and PsA from the Swiss Clinical Quality Management register (SCQM) using a smartphone app (mySCQM) to record information between March and December 2020. The outcomes of interest were self-reported SARS-CoV2 testing, symptoms compatible with COVID-19 during the previous month and confirmed COVID-19 through PCR nasopharyngeal swab. Outcomes were evaluated over the complete length of the aforementioned period (i.e. the outcome has been reported at least once during the period). Outcomes were compared between diseases groups, using logistic regression. We also evaluated the association of baseline treatment (TNF-inhibitors, b/tsDMARDs with other modes of action (OMA), no b/tsDMARDs) on the odds of symptoms and testing and the association of the number of symptoms (0-9) on the odds of testing. The analyses of SARS-CoV2 testing and COVID-19 symptoms were additionally adjusted for age, gender, glucocorticoids and csDMARDs. Confirmed cases were not adjusted for treatment and other covariates considering the low number of events.

Results: We included 927 patients with RA, 805 with AxSpa and 453 with PsA (Table 1). 1010 patients reported COVID-19-like symptoms (mostly fever, runny nose and cough), but only 455 of them (45\%) reported being tested. 151 patients were tested without symptoms. In between March and December 2020, 7.6\% of RA, $8.5 \%$ of AxSpA and $10.5 \%$ of PsA patients were tested positive for COVID-19 $(p=0.678)$. The odds of testing, symptoms and confirmed COVID-19 were similar between diseases and not associated with treatment for testing and symptoms (Figure 1). The number of symptoms was associated with the odds of testing (OR $1.43,95 \% \mathrm{Cl} 1.37-1.50$ by symptom)

Table 1.

\begin{tabular}{lccccc}
\hline & & RA & axSpA & PsA & p \\
\hline $\mathrm{n}$ & & 927 & 805 & 453 & \\
Age (mean, SD) & & $56.4(13)$ & $47.1(12)$ & $52.7(11)$ & $<0.001$ \\
Gender & female & $705(76)$ & $403(50)$ & $230(51)$ & $<0.001$ \\
Treatment & TNFi & $272(29)$ & $498(62)$ & $174(38)$ & $<0.001$ \\
& OMA & $355(38)$ & $71(9)$ & $137(30)$ & \\
& No b/tsDMARDs & $300(32)$ & $236(29)$ & $142(31)$ & \\
csDMARDs use & & $476(51)$ & $111(14)$ & $147(33)$ & $<0.001$ \\
Steroids use & & $93(10)$ & $11(1)$ & $19(4)$ & $<0.001$ \\
Disease duration & & $14.2(10)$ & $17.4(11)$ & $14.8(12)$ & $<0.001$ \\
Testing for COVID-19 & All patients & $258(28)$ & $231(29)$ & $117(26)$ & 0.550 \\
& Patients with & $189 / 427(44)$ & $179 / 392(46)$ & $87 / 191(46)$ & 0.911 \\
& symptoms & & & & \\
Presence of COVID-19 & & $427(46)$ & $392(49)$ & $191(42)$ & 0.082 \\
$\quad$ symptoms & & $18 / 237(8)$ & $19 / 223(9)$ & $11 / 105(11)$ & 0.678 \\
Positive COVID-19 test & & & &
\end{tabular}

$\mathrm{N}, \%$ when not specified otherwise. ${ }^{*}$ Missing data on test results in 41 patients $\chi 2$ test for categorical and t-test for continuous variables.

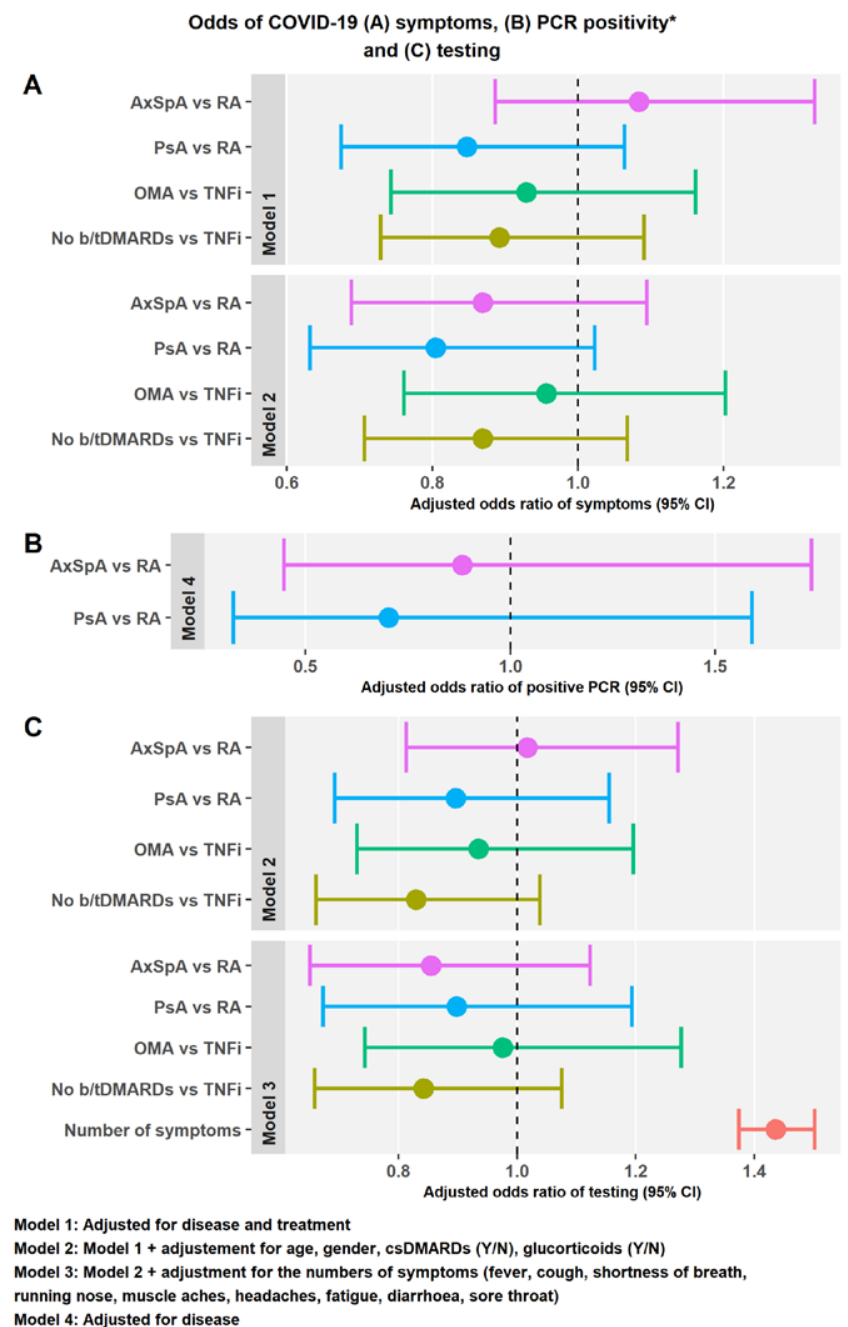

*In patients with information on tests results ( $\mathrm{N}=565$

Figure 1. 